\title{
Amorphous silica between confining walls and under shear: a computer simulation study
}

\author{
Jürgen Horbach and Kurt Binder \\ Institut für Physik, Johannes Gutenberg-Universität, Staudinger Weg 7, D-55099 Mainz, \\ Germany
}

\begin{abstract}
Molecular dynamics computer simulations are used to investigate a silica melt confined between walls at equilibrium and in a steady-state Poisseuille flow. The walls consist of point particles forming a rigid face-centered cubic lattice and the interaction of the walls with the melt atoms is modelled such that the wall particles have only a weak bonding to those in the melt, i.e. much weaker than the covalent bonding of a $\mathrm{Si}-\mathrm{O}$ unit. We observe a pronounced layering of the melt near the walls. This layering, as seen in the total density profile, has a very irregular character which can be attributed to a preferred orientational ordering of $\mathrm{SiO}_{4}$ tetrahedra near the wall. On intermediate length scales, the structure of the melt at the walls can be well distinguished from that of the bulk by means of the ring size distribution. Whereas essentially no structural changes occur in the bulk under the influence of the shear fields considered, strong structural rearrangements in the ring size distribution are present at the walls as far as there is a slip motion. For the sheared system, parabolic velocity profiles are found in the bulk region as expected from hydrodynamics and the values for the shear viscosity as extracted from those profiles are in good agreement with those obtained in pure bulk simulations from the appropriate Green-Kubo formula.
\end{abstract}

\section{INTRODUCTION}

The transport properties of atomic fluids that move through nanoscopic slits between solid walls has been an issue of constant interest over the past decades [1 simulation studies investigating such fluids in Couette or Poisseuille flow have considered simple monoatomic systems in which the particles interact via Lennard-Jones (LJ) type forces (see, e.g., [6 9]). One of the remarkable results of these studies was that velocity profiles as predicted by hydrodynamics can be observed even if the distance between the confining walls is of the order of only ten molecular diameters. But one has to keep in mind that the monoatomic LJ systems for which this was found have typically a relatively small viscosity (of the order of $10^{-4}$ to $10^{-3}$ Poise in Argon units) and furthermore, these systems crystallize very easily if one decreases the temperature below the melting point. An open question is what happens with more complicated sheared fluids that are confined between walls, i.e. especially liquids that have a structure that is not similar to the local structure 
of a closed-packed system of hard spheres like the aforementioned LJ systems. One class of more complex materials for which flow properties in confinement have already been studied to some extent are polymer melts [12 [18]. Very recently it was shown [18] for such a system that its properties under shear can be well understood within a hydrodynamic description.

In this paper we are interested in amorphous silica $\left(\mathrm{SiO}_{2}\right)$ which is the paradigm of a network forming liquid. We consider a $\mathrm{SiO}_{2}$ melt that is confined between two crystalline walls and on which a gravitational-like field is exerted in order to yield a stationary Poisseuille flow. It is difficult to address this problem in a simulation because model potentials of silica contain long-ranged Coulomb terms [19] for which the corresponding force calculations become very time-consuming for a three dimensional system in which periodic boundary conditions are considered only in two directions. This stems from the fact that the Fourier part of the Ewald sums can no longer be calculated by a single loop over the number of particles $N$ as in the case of periodic boundary conditions in three dimensions, but one has to compute a double loop that scales with $N^{2}$ [20 23]. Therefore, very recently, many efforts were undertaken to develop alternative methods 24 27] for the summation of "twodimensional" Coulomb interactions. But most of these new approaches are not established yet and extensive tests that demonstrate that their accuracy is comparable to the "brute force" $N^{2}$-methods are still lacking. Moreover, differently from monoatomic LJ systems, silica is a good glassformer that exhibits a slow dynamics even above the melting temperature. Note that at the experimental melting temperature, $T=2000 \mathrm{~K}$, amorphous silica has a viscosity of about $10^{7}$ Poise [28]. Systems with such a high viscosity cannot be equilibrated nowadays by means of a molecular dynamics computer simulation.

The structure of silica is that of a disordered tetrahedral network in which $\mathrm{SiO}_{4}$ tetrahedra are connected to each other in that they share an oxygen atom at their corner. Extensive simulation studies of free silica surfaces, that used both classical molecular dynamics as well as Car-Parrinello simulations [30,31] gave evidence for a different packing of the $\mathrm{SiO}_{4}$ tetrahedra at the surface from that in the bulk. This different packing is manifested very clearly in the ring structure (a ring is a closed loop formed by consecutive $\mathrm{Si}-\mathrm{O}$ elements). At the surface there is a relatively high probability to find short rings such as two- and three-membered rings whereas in the bulk the occurence of such rings is very rare even at relatively high temperatures. Note that the occurrence of short rings on a free silica surface has been confirmed experimentally by means of atomic force microscopy [32]. A detailed understanding of the structure near the wall is important to give insight into the microscopic origin of the wall-fluid boundary conditions if a flow field is introduced. This might be especially important in the case of silica since it does not form a closed-packed structure and thus the behavior could be very different from that of a hard-sphere like system. Indeed we show below that there is a rearrangement in the ring distribution near the walls if the shear is strong enough.

An important issue for the understanding of a confined fluid under shear are the boundary conditions that enter in the hydrodynamic description in order to take into account the interactions of the fluid with the wall. In principle, these boundary conditions can be derived from the microscopic equations of motion for the fluid and the wall particles. But in practice one often introduces as an ad hoc assumption stick boundary conditions. However, it has been found in simulations of simple fluids that there is often a slip motion of the fluid at the wall [10,[1]]. So in general one has to consider slip-stick boundary conditions 
for a hydrodynamic analysis of fluid flow between walls. Bocquet and Barrat 33 have shown in a microscopic theory, in which they have related quantities like the slip length with equilibrium time correlation functions via Green-Kubo formulas, that slip occurs if the walls do not provide a strong corrugation. E.g., a large slip effect is observed if the fluid partially wets the solid walls. In a recent simulation of Sokhan et al. [34 it has been even demonstrated for the fluid flow through a carbon tube that slip-stick boundary conditions are the generic case if one uses realistic parameters in the model potential for the wallfluid interactions. It is one of the issues of this paper to characterize the structure of the silica melt near the wall and to see how the structure is related to the observed boundary conditions.

Another issue that we address in this paper is to discuss to what extent the velocity profiles from our simulations can be used to estimate the shear viscosity $\eta$ of a highly viscous fluid such as silica. An alternative method is to calculate $\eta$ from the appropriate GreenKubo formula [35] which expresses $\eta$ as the time integral over a stress-stress autocorrelation function. We applied the latter approach recently in extensive bulk simulations of silica in which we were able to determine $\eta$ in the temperature range $6100 \mathrm{~K} \geq T \geq 3000 \mathrm{~K}$ whereby at $T=3000 \mathrm{~K}$ the visosity is of the order of 60 Poise [36]. In the present paper we use the latter results as a reference to see how reliable the estimates of $\eta$ from our non-equilibrium simulations are. Such a reference is important since it is not obvious that the observed velocity profiles justify a "hydrodynamic analysis" keeping in mind that the distance between the confining walls is on a nanoscopic scale.

The rest of the paper is organized as follows: In Sec. II we describe how we model the walls and the silica melt, and we give the details of the simulation. Sec. III is then devoted to the presentation of the results and eventually, in Sec. IV we summarize and discuss them.

\section{MODEL AND DETAILS OF THE SIMULATION}

A realistic simulation of silica confined between walls requires an interaction potential that yields a reliable description of both the bulk properties and the properties at the interface between the walls and the silica fluid. As far as the bulk properties are concerned recent simulations have shown that the potential that was proposed some years ago by van Beest et al. 37] (the so-called BKS potential) is able to reproduce various properties of amorphous silica very well, such as its structure, its vibrational and relaxational dynamics, the static specific heat below the glass transition temperature, and the conduction of heat [36, 38, 45]. Recent extensive simulations [31] that used a combination of a classical molecular dynamics simulation with the BKS potential and ab initio simulations (Car-Parrinello molecular dynamics) gave evidence that the BKS potential yields also a fair description of the structure of free amorphous silica surfaces, and thus, it can be expected that it also reproduces the main features of the structure of amorphous silica near a wall.

The functional form of the BKS potential is given by

$$
\phi_{\alpha \beta}(r)=\frac{q_{\alpha} q_{\beta} e^{2}}{r}+A_{\alpha \beta} \exp \left(-B_{\alpha \beta} r\right)-\frac{C_{\alpha \beta}}{r^{6}} \quad \alpha, \beta \in[\mathrm{Si}, \mathrm{O}]
$$

where $r$ is the distance between the ions of type $\alpha$ and $\beta$. The values of the constants $q_{\alpha}, q_{\beta}, A_{\alpha \beta}, B_{\alpha \beta}$, and $C_{\alpha \beta}$ can be found in Ref. [37]. For the sake of computational efficiency 
the short range part of the potential was truncated and shifted at $5.5 \AA$. This truncation has also the benefit to improve the agreement between the density of the amorphous glass at low temperatures as determined from the simulation with the experimental value. For the calculation of the long-ranged Coulomb interactions we used the method first introduced by Parry [20,21] which is a straightforward generalization of the three-dimensional Ewald sums with periodic boundary conditions in all three directions. In the latter case the Fourier sum requires only a loop over the $N$ particles of the system which leads, together with the real space sum, to an effective computational load that scales as $N^{1.5}$ [29]. In contrast to that in the Parry-Ewald sums for systems with a quasi-two-dimensional geometry the Fourier and the real space part have to be computed by a double loop over all pairs of particles. However, we preferred to use Parry-Ewald sums rather than alternative faster methods [24 27] since it provides accurate results and it is easy to implement.

The walls were not constructed as to model a particular material but rather to be a generic surface that can be simulated conveniently. Each wall hence consisted of 563 point particles forming a rigid face-centered cubic lattice with a nearest-neighbor distance of $2.33 \AA$. These point particles interact with the atoms in the fluid according to a $12-10$ potential,

$$
v(r)=4 \epsilon\left[(\sigma / r)^{12}-(\sigma / r)^{10}\right],
$$

with $\sigma=2.1 \AA, \epsilon=1.25 \mathrm{eV}, r$ being the distance between a wall particle and a Si or O atom. Note that we choose the same potential between the wall atoms and both $\mathrm{Si}$ and O because we wish to simulate the generic effects of confinement, separated from possible additional effects due to surface enrichment of one component caused by the difference in surface forces, which may be a problem in many real systems. The great advantage of simulation is that by idealizing certain aspects of a problem a much better physical understanding of consequences of particular interactions may be achieved. In this spirit, a wall potential has been "invented" that keeps the slip small (when we apply a constant force in $x$-direction to the particles causing a corresponding flow) and provides at the same time a weak bonding of the wall particles to those of the melt such that no covalent bonds of wall particles with $\mathrm{Si}$ or $\mathrm{O}$ atoms are formed. In order fulfil these two "requirements" the $r^{-10}$-term in Eq. (2) has turned out to be more suitable than a $r^{-6}$-term.

The simulation box had linear dimensions $L_{x}=L_{y}=23.066 \AA$ in the directions parallel to the walls (in which also periodic boundary conditions were applied), and $L_{z}=31.5 \AA$ in the direction perpendicular to the walls. Thus, $N=1152$ atoms (384 Si atoms and $768 \mathrm{O}$ atoms) were contained in the system to maintain a density around $2.3 \mathrm{~g} / \mathrm{cm}^{3}$ which is close to the experimental one at zero pressure [28]. In the Parry-Ewald sums the parameter $\alpha$ and the cutoff wave-vector for the Fourier part were chosen to 0.265 and 6, respectively [29. The equations of motion were integrated with the velocity form of the Verlet algorithm with a time step of $1.6 \mathrm{fs}$. All runs were done in the NVT ensemble whereby the temperature was kept constant by coupling the fluid to a Nosé-Hoover thermostat 46 48. Whenever an external gravitational-like field was switched on, the thermostat was only applied in the two directions perpendicular to the flow, i.e. in $y$-and $z$-direction, whereas otherwise the thermostat was used in all three directions. Bocquet and Barrat [11] also embarked on this strategy to provide a correct adjustment of the temperature when the system is sheared.

We investigated the three temperatures $T=5200 \mathrm{~K}, T=4300 \mathrm{~K}$, and $T=3760 \mathrm{~K}$ at 
which we first fully equilibrated the system for $29 \mathrm{ps}, 65 \mathrm{ps}$, and $122 \mathrm{ps,}$ respectively. At $T=5200 \mathrm{~K}$ and $T=3760 \mathrm{~K}$ we continued with additional runs over $164 \mathrm{ps}$ and $490 \mathrm{ps}$, respectively, from which we analyzed the equilibrium structure. Then we switched on a gravitational acceleration field of strength $a_{\mathrm{e}}=9.6 \AA / \mathrm{ps}^{2}$ that was coupled to the mass of the particles. We mention that although the masses of oxygen and silicon differ by about a factor of two $\left(m_{\mathrm{O}}=15.9994 \mathrm{u}, m_{\mathrm{Si}}=28.086 \mathrm{u}\right)$ we obtained identical velocity profiles for both species (see below), and this is due to the fact that the chemical bonding force between oxygen and silicon atoms is much stronger than the imposed acceleration field. With the acceleration field runs were made over $736 \mathrm{ps}, 1.23 \mathrm{~ns}$, and $3.27 \mathrm{~ns}$ at $T=5200 \mathrm{~K}, 4300 \mathrm{~K}$, and $3760 \mathrm{~K}$, respectively. In addition at $T=5200 \mathrm{~K}$ we did a run over $1.72 \mathrm{~ns}$ with field strength $a_{\mathrm{e}}=3.8 \AA / \mathrm{ps}^{2}$. During these runs we stored the positions and velocities of the particles every 0.16 ps (i.e. every 100 time steps). For the calculation of the steady state properties we used only those data that were obtained after two times the time that was used before for the equilibration. The total amount of computer time spent for these simulations was 16 years of single processor time on a Cray T3E.

\section{RESULTS}

In this section we present the simulation results for the silica melt between confining walls. We characterize the structure of the melt and show how it changes if one switches on a gravitational field, i.e., if a Poisseuille flow is present in the melt. By analyzing the velocity profiles we discuss to what extent their behavior can be understood within a hydrodynamic description.

The snapshot, Fig. 1, illustrates on what length scales the walls affect the structure of the melt. It shows a part of a configuration at $T=3760 \mathrm{~K}$, namely the part that is within a distance less than $8 \AA$ away from one of the walls. One can clearly identify a layering of $\mathrm{SiO}_{4}$ tetrahedra which becomes less pronounced if one moves away from the wall. Note that at $T=3760 \mathrm{~K} 95 \%$ of the silicon atoms are four-fold coordinated by oxygen atoms and $96.5 \%$ of the oxygen atoms are two-fold coordinated by silicon atoms (a silicon and an oxygen atom are defined as neighbors if their distance is less than $2.35 \AA$ corresponding to the first minimum of the pair correlation function for $\mathrm{Si}-\mathrm{O}$ ). The rest of the atoms form defects, i.e. silicon atoms that are three- or five-fold coordinated by oxygen atoms and oxygen atoms that are one- or three-fold coordinated by silicon atoms. Thus, since less than $5 \%$ of the atoms are involved in such defects it makes sense to characterize the structure also at the relatively high temperature $T=3760 \mathrm{~K}$ as a disordered tetrahedral network where $\mathrm{SiO}_{4}$ tetrahedra are connected via bridging oxygens (i.e. every oxygen atom belongs to two tetrahedra).

The layering of the $\mathrm{SiO}_{4}$ network near the wall can be quantified by means of the density profile which is plotted in Fig. 2a across half of the film 49 for all atoms and for the oxygen and silicon atoms only. In contrast to typical density profiles in simple monoatomic liquids the oscillations of the total profile, which indicate the layering near the walls, do not have a regular character. But this irregular behavior can be understood if one looks at the partial profiles for oxygen and silicon. The explanation that emerges is as follows: As we mentioned before confined fluid $\mathrm{SiO}_{2}$ maintains the irregular network $\mathrm{SiO}_{4}$ tetrahedra as in the bulk. At the same time the tetrahedra have to fill the available space and have to satisfy the bonding 
energy to the wall. To this end, it is necessary to align the tetrahedra adjacent to the wall such that a two-dimensional plane forms which contains three out of the four oxygen atoms of a tetrahedron, as well as the silicon atom at their center (slightly further away from the wall), while the fourth oxygen atom of the tetrahedron has to be further away from the wall for geometrical reasons: this causes the second peak of the oxygen distribution. Thus, the walls have a tendency to "orient" the network of coupled $\mathrm{SiO}_{4}$ tetrahedra in the fluid locally. It is evident that the oscillations in the local density of both silicon and oxygen are rather regular, like a damped cosine function, but the wavelength and phase of both cosine functions are different: their superposition causes then the rather irregular layering structure of the $\mathrm{SiO}_{2}$ total density. We expect that similar effects also occur in many other associating molecular fluids confined between walls if the wall-fluid interaction is weak enough that it does not affect the chemical ordering in the fluid as it is the case in our system.

For $z>8 \AA$ the total density profile shows only small oscillations around a constant value of $2.3 \mathrm{~g} / \mathrm{cm}^{3}$ which is an indication for bulk behavior. Thus, keeping in mind that the density profile is symmetric, the bulk in our system seems to extend in $z$ direction from about $8 \AA$ to $23.5 \AA$, i.e. it has a width of about $15.5 \AA$. However, Fig. 2 a shows that the deviations from a constant density in the latter region are not due to the statistics: The partial density profiles exhibit still oscillations also in the middle of the film that one would not expect in a pure isotropic bulk system. But these oscillations do not affect the structural quantities that we show below, i.e. the pair correlation functions and the distributions of angles and rings, since these quantities essentially do not differ from the corresponding ones of a pure bulk simulation at the same temperature. Therefore, we consider in the following the region defined by $8 \AA \leq z \leq 23.5 \AA$ as the bulk.

The behavior of the total density profiles at the temperatures $T=5200 \mathrm{~K}$ and $T=$ $3760 \mathrm{~K}$ in equilibrium and with an external force with an acceleration of $9.6 \AA / \mathrm{ps}^{2}$ can be seen in Fig. 2 $\mathrm{b}$. In equilibrium the effect of decreasing temperature on the oscillations near the wall is an increase of the peak heights that is accompanied with a smaller value of $\rho(z)$ at the minima between the peaks. Thus, the layering becomes more pronounced if one decreases the temperature. If one switches on the gravitational-like field the effect is similar to an increase of the temperature. In the bulk region the density profiles are not very sensitive to a variation of temperature and/or the presence of the external force. Within the accuracy of our data the same value of about $2.3 \mathrm{~g} / \mathrm{cm}^{3}$ is reached for all four cases under consideration. However, this does not exclude of course the possibility of a dramatic change in the local structure, but as we will see in the following, the local structure is not strongly affected by the considered external forces.

Quantitities that provide information on the microscopic interparticle distances are the partial pair correlation functions $g_{\alpha \beta}(r)$ which are proportional to the probability of finding an atom of type $\alpha$ at a distance $r$ from an atom of type $\beta$. In an isotropic system the functions $g_{\alpha \beta}(r)$ are normalized by a phase factor $4 \pi r^{2}$ 35. However, for the case that boundaries are present this factor has to be modified in that it is then determined only by that part of the surface $4 \pi r^{2}$ of a sphere around a particle that fits into the system. Thus, the normalization factor depends on the distance of the particles from the walls in $z$-direction. In order to identify the differences of $g_{\alpha \beta}(r)$ close to the wall from those of the bulk we define a bulk region for $8 \AA \leq z \leq 23.5 \AA$ and wall regions for distances less or equal $3 \AA$ from the wall (containing respectively the first oxygen and silicon layer, see Fig. 2a). 
The latter wall layer is denoted in the following by WL. The $g_{\alpha \beta}(r)$ are calculated only for particles within the bulk or the WL whereby the aforementioned normalization factors are determined such that also the boundaries of the bulk region and the WL that are located within the fluid film are treated as real boundaries. Fig. 3 shows $g_{\alpha \beta}(r)$ for the different correlations at $T=3760 \mathrm{~K}$, each at equilibrium in the bulk and in the $\mathrm{WL}$, as well as in the WL under the acceleration field $a_{\mathrm{e}}=9.6 \AA / \mathrm{ps}^{2}$. We have not included the curves for the bulk with an external field because they essentially coincide with the equilibrium curves. This means that for the field strength $a_{\mathrm{e}}=9.6 \AA / \mathrm{ps}^{2}$ the structure as reflected in $g_{\alpha \beta}(r)$ is not affected in the bulk by the gravitational field. From this we can consider the latter field strength as a small disturbance, and thus we can expect that the system can be treated as a Newtonian fluid in the bulk. In contrast to this at the walls there are significant changes in $g_{\alpha \beta}(r)$ if one switches on the external field in that the peaks become broader and their heights decrease slightly. These changes are most pronounced in $g_{\mathrm{OO}}(r)$ which is reasonable since the very first layer at the wall is formed by oxygen atoms.

The differences in $g_{\alpha \beta}(r)$ at the WL and in the bulk reflect the different packing of the tetrahedral network in both regions. In $g_{\mathrm{SiO}}(r)$ the second and third peaks are shifted to smaller distances in the bulk as compared to the WL. Similar effects are even more pronounced in $g_{\mathrm{OO}}(r)$ and $g_{\mathrm{SiSi}}(r)$. In these functions one can see that the fcc lattice of the wall affects the structure on length scales of next-nearest $\mathrm{Si}-\mathrm{Si}$ and $\mathrm{O}-\mathrm{O}$ neighbors. In $g_{\mathrm{OO}}(r)$ for the WL the periodicity of the peaks is approximately given by the lattice constant $a=2.33 \AA$ of the fcc lattice that is formed by the wall atoms. This periodicity appears less pronounced in $g_{\mathrm{SiSi}}(r)$ since the $\mathrm{Si}$ atoms are not so close to the wall as the $\mathrm{O}$ atoms and have thus more freedom to arrange themselves. Moreover the first peak in $g_{\mathrm{SiSi}}(r)$ is at a slightly smaller distance for the WL than for the bulk and this is due to the denser packing of $\mathrm{SiO}_{4}$ tetrahedra near the wall.

A further step towards the characterization of the local structure is to consider correlations between triples of particles. Simple quantities to study such correlations are the distributions of angles between three neighboring atoms. Thereby, two atoms of type $\alpha$ and $\beta$ are defined as neighbors if their distance is smaller than the position of the first minimum in the corresponding $g_{\alpha \beta}(r)$. The locations of these minima are at $3.64 \AA, 2.35 \AA$, and $3.21 \AA$ for the $\mathrm{Si}-\mathrm{Si}, \mathrm{Si}-\mathrm{O}$, and $\mathrm{O}-\mathrm{O}$ correlations, respectively. In Fig. 四 we show the angle distribution functions $P_{\alpha \beta \gamma}(\theta)$ for the $\mathrm{Si}-\mathrm{Si}-\mathrm{Si}, \mathrm{Si}-\mathrm{O}-\mathrm{Si}$, and the $\mathrm{O}-\mathrm{Si}-\mathrm{O}$ angles in the bulk at equilibrium and under shear and at the WL at equilibrium. The temperature in all cases is $3760 \mathrm{~K}$. We see in the figure that in each of the $P_{\alpha \beta \gamma}(\theta)$ shown there are only small differences between the bulk curves for the equilibrium and the sheared case which again demonstrates that the structure changes only slightly in the bulk region if a field of strength $a_{\mathrm{e}}=9.6 \AA / \mathrm{ps}^{2}$ is switched on. There are also only small differences in $P_{\mathrm{OSiO}}$ for the WL as compared to the corresponding distributions in the bulk. Since the $\mathrm{O}-\mathrm{Si}-\mathrm{O}$ angle is an intratetrahedral one this shows that the geometry of the $\mathrm{SiO}_{4}$ tetrahedra is essentially the same at the wall and in the bulk. Remarkable differences are in $P_{\mathrm{SiSiSi}}$ and $P_{\mathrm{SiOSi}}$ for the WL as compared to corresponding functions for the bulk: First, the main peak in $P_{\mathrm{SiOSi}}$ is shifted towards smaller angles which is due to the denser packing of the tetrahedra in the WL. Secondly, there is a shoulder in $P_{\mathrm{SiOSi}}$ around $100^{\circ}$ which is less pronounced in the WL and $P_{\mathrm{SiSiSi}}$ exhibits a secondary peak at about $60^{\circ}$ which is more pronounced in the curve for the WL. In Fig. Gb we compare the different $P_{\alpha \beta \gamma}(\theta)$ for the WL at equilibrium and for 
the sheared system. As can be inferred from the figure, in contrast to the bulk case there are changes in the angular distributions in that all three distributions broaden significantly for the system under shear. Moreover, the shoulder in $P_{\mathrm{SiOSi}}$ around $100^{\circ}$ has about a six times larger amplitude in the latter case.

Recent studies of silica melts [30,31] have shown that the shoulder around $100^{\circ}$ in $P_{\mathrm{SiOSi}}$ is due to two-membered rings whereas the peak at about $60^{\circ}$ in $P_{\mathrm{SiSiSi}}$ corresponds to the presence of three-membered rings. We look now for the latter ring sizes in our system and investigate the distribution of rings in the network. A ring is defined as follows: One starts from any silicon atom and two of its nearest oxygen neighbors. Then one counts the shortest consecutive sequence of $\mathrm{Si}-\mathrm{O}$ elements that connect the two latter oxygen atoms and this number is then the ring length $n$. Thereby, the ring length $n=1$ corresponds to a dangling bond, i.e., an $\mathrm{O}$ atom is only attached to one Si atom, and in a two-membered ring $(n=2)$ two silicon atoms share two oxygens. Note that one can extract (indirectly) information on rings in $\mathrm{SiO}_{2}$ by means of NMR experiments [50].

Fig. 5a shows the ring distribution function $P(n)$ for $T=3760 \mathrm{~K}$ in the bulk and in two different wall layers denoted by WL1 and WL2. WL1 and WL2 are defined as the regions which are respectively within a distance of $6.25 \AA$ and $3.0 \AA$ away from the wall corresponding to the second minimum of the density profile for silicon and the first minimum of the total density profile, respectively (see Fig. 2a). Note that WL2 is identical with WL. In each region, i.e. bulk, WL1, and WL2, we took only those rings into account that fit completely into it. Thus, in WL2 those rings are counted that are formed at each case by the first and the second $\mathrm{O}$ and $\mathrm{Si}$ layers (with respect to the distance from the wall), whereas with WL2 only those rings are taken into account that are formed by the first $\mathrm{O}$ and the first Si layer. This is justified because the first two oxygen and the first two silicon layers are well-defined in that the minima in the corresponding density profiles are close to zero density in the case of WL2 and around the small value $\rho=0.5 \mathrm{~g} / \mathrm{cm}^{3}$ in the case of WL1. Furthermore, we can infer from Fig. Aa that in contrast to the second layers the first oxygen layer overlaps strongly with the first silicon layer and the overall thickness of both layers is only about $2 \AA$. Thus, the first oxygen and the first silicon layer form a quasi-twodimensional plane and $P(n)$ for WL2 gives a distribution of rings that have an orientation parallel to the walls whereas in $P(n)$ for WL1 also the rings perpendicular to the walls are included.

As has been also found in pure bulk simulations of $\mathrm{SiO}_{2}$ [38, in the bulk a maximum is observed around $n=6$. This is plausible since in silica the high-temperature crystalline phase at zero pressure, $\beta$-cristobalite, exhibits only six-membered rings. In WL1 the probability for $n \geq 6$ is smaller than in the bulk in favor of a relatively high probability of $n=3$ and $n=4$. In WL2 it is the other way round: $n=4$ and even more $n=5$ are less frequent than in the bulk in favor of $n=8,9,10$. The ring structure near the walls that corresponds to these findings is as follows: Perpendicular to the walls small rings with $n=3,4$ are seen such that, e.g., $n=3$ is formed by two silicon atoms from the first silicon layer with a third one from the second silicon layer (for an illustration see Fig. 1). In contrast to that, parallel to the walls (considering the first oxygen and the first silicon layer) an open structure with relatively large rings is observed which compensates somewhat the dense packing of $\mathrm{SiO}_{4}$ tetrahedra perpendicular to the walls.

Fig. 5b shows the behavior of $P(n)$ in the bulk at the two temperatures $T=3760 \mathrm{~K}$ 
and $T=5200 \mathrm{~K}$ in equilibrium and under shear. We can immediately infer from the figure that the considered shear fields have only a small effect on the structure which confirms the findings for all the quantities discussed before. At $T=5200 \mathrm{~K}$ one has a relatively large amount of two- and three-membered rings the frequency of which is more than a factor of two smaller at $T=3760 \mathrm{~K}$. In Ref. [38] it was shown that their frequency of occurrence decreases further with decreasing temperature such that the amount of two-membered rings falls far below $1 \%$ for systems that have typical structural relaxation times of the order of 1 ns.

In contrast to the bulk in WL1 significant changes in the ring distribution take place if the system is sheared (see Fig. 5c), and the external force field affects the ring structure such that small and large rings are formed, while at the same time especially the amount of sixmembered rings decreases. Only for the smaller field strength $a_{\mathrm{e}}=3.8 \AA / \mathrm{ps}^{2}$ there are no significant changes in the ring distribution. We will see below that the latter is accompanied with a very small slip motion at the walls whereas a large slip velocity is correlated with strong rearrangements in the ring structure. One can also infer the remarkable fact from Fig. 50 that the probability to find rings with $n=3,4$ does not change very much when an external acceleration field is switched on. This is reasonable because these small-membered rings, as we have seen before, are located perpendicular to the walls and thus they are very stable to shear forces that are imposed parallel to the walls.

The strongest rearrangements in the ring structure due to a shear field are found when we consider the region WL2. The corresponding curves are shown in Fig. 55d. Again, there are only minor changes in $P(n)$ at $T=5200 \mathrm{~K}$ and $a_{\mathrm{e}}=3.8 \AA / \mathrm{ps}^{2}$ as compared to the corresponding equilibrium case which is, as mentioned before, related to the presence of only a very small slip velocity. For the higher acceleration field, $a_{\mathrm{e}}=9.6 \AA / \mathrm{ps}^{2}$, the ring structure becomes more heterogeneous and the effect of the external field is if one would locally increase the temperature. The rearrangements in the ring structure can be summarized as follows: Rings mainly of size $n=6,7,8$ are broken under the influence of the shear force and instead small rings with $n<4$ and very large rings with $n \geq 9$ are formed. This is illustrated in Fig. [ 0 where snapshots at $T=3760 \mathrm{~K}$ of the WL2 region are shown for the equilibrium case and the non-equilibrium steady state case with the external field of strength $a_{\mathrm{e}}=9.6 \AA / \mathrm{ps}^{2}$. One can clearly identify, e.g., rings with $n=2$ and $n=11$ in the right panel which are absent in the left panel, i.e. at equilibrium.

Up to now we have seen that in the bulk region the structure essentially does not change for acceleration fields $a_{\mathrm{e}} \leq 9.6 \AA / \mathrm{ps}^{2}$ whereas significant structural rearrangements occur close to the walls for $a_{\mathrm{e}}=9.6 \AA / \mathrm{ps}^{2}$. We demonstrate now how this is reflected in the flow properties, and we discuss to what extent the flow can be understood within a hydrodynamic description.

Fig. 7 shows velocity profiles $v_{x}(z)$ across the film for two different choices of the acceleration in $x$-direction for $T=5200 \mathrm{~K}$. The solid lines are fits according to the formula

$$
v_{x}(z)=\frac{\rho a_{\mathrm{e}}}{2 \eta}\left(z-z_{1}\right)\left(L_{z}-z_{1}-z\right)
$$

with $\rho$ being the total mass density, and the intercept $z_{1}$ as well as the shear viscosity $\eta$ are treated as adjustable parameters. Eq. (3) is the formula for a one-component fluid, but it is justified to use this equation for our two-component system since the velocity profiles for the individual species, i.e. for silicon and oxygen, are identical within the statistical 
errors. Note that this was also observed by Koplik and Banavar [51] for a mixture of two simple liquids confined between two walls. The values of $\eta$ that result from the fits with Eq. (3) are quoted in the figure. As it should be, $\eta$ is independent of the acceleration $a_{\mathrm{e}}$ within statistical errors. This matches well to the aforementioned result that the structure is essentially not affected in the bulk by the considered shear fields. Furthermore, this analysis shows that hydrodynamics holds down to nanoscopic scales, even if we use a realistic model of a molecular glassforming fluid such as $\mathrm{SiO}_{2}$. Close to the wall the fit with Eq. (3) is obviously not valid for $a_{\mathrm{e}}=9.6 \AA / \mathrm{ps}^{2}$ and we find a slip motion of the fluid. As we have seen before this slip is accompanied with structural rearrangements in the ring distribution.

It is conspicuous in Fig. 7 that there is a sharp peak in $v_{x}(z)$ at $z=1.2 \AA$ in the curve for $a_{\mathrm{e}}=3.8 \AA / \mathrm{ps}^{2}$ (there is exactly the same peak at $z=30.3 \AA$ because the profile is symmetrized). This is just due to the fact that there is a very small probability for the particles to move into the region $z<1.5 \AA$, i.e. the region very close to the wall. And if a particle enters this region there is a high probability that it has a relatively high velocity. Of course, on average this velocity should be zero, but in this case the statistics is bad since this event is very rare, and we observe sharp peaks in the regions very close to the walls that can have a negative or positive amplitude with equal probability (note that they appear also in the unsheared system). A similar reasoning might be also true at $z=3 \AA$, i.e. at the location of the first minimum in the total density profile. Indeed we see a small peak at $z=3 \AA$ in the curve for $a_{\mathrm{e}}=9.6 \AA / \mathrm{ps}^{2}$ which we also find at lower temperatures (see Fig. 8). But we cannot conclude from our data whether this feature would disappear with a better statistics.

One still could argue that the fits with Eq. (3) are fortuitous, and therefore the resulting shear viscosity $\eta$ then simply is a fit parameter with little physical significance, different from the actual shear viscosity of bulk molten $\mathrm{SiO}_{2}$. Fig. 8 shows that this objection is not true: here the velocity profiles are compared at three temperatures, and the viscosities $\eta$ from the fits are compared to viscosities $\eta_{\text {GK }}$ obtained earlier from simulations of bulk $\mathrm{SiO}_{2}$ at rest (no walls, no flow) applying Green-Kubo formulas [36]. Note that the latter approach typically involves a relative error of about $20 \%$, and so does the present approach. Within these errors, there is a good agreement, although $\eta_{\mathrm{GK}}$ changes for the shown range of temperatures by an order of magnitude. However, the estimated $\eta$ seems to be systematically smaller than $\eta_{\text {GK }}$ at low temperatures and we cannot exclude that these deviations are due to the influence of the Nosé-Hoover thermostat on the flow velocity. Of course, we can also infer from Fig. 8 that we cannot use our simulation of Poisseuille flow to estimate the viscosity at much lower temperatures than $3760 \mathrm{~K}$ : If $\eta$ decreases the velocity profile becomes flatter, and one cannot compensate this by higher values of $a_{\mathrm{e}}$ since the slip velocity increases with $a_{\mathrm{e}}$ also.

\section{SUMMARY AND DISCUSSION}

We used molecular dynamics computer simulations to study a silica melt confined between two walls. The interactions between the silicon and oxygen atoms were modelled by the so-called BKS potential for which it has been shown that it describes reliably the bulk as well as free surface properties of silica. The walls consist of particles fixed on a fcc lattice, and wall-fluid interactions were modelled by a simple 12-10 potential. The aim of this work was to investigate how the walls affect the structure of the melt in equilibrium and under 
shear, and whether it is possible to understand the flow properties of the sheared melt within hydrodynamics.

As in the case of simple liquids a pronounced layering of the melt close to the walls is observed. This layering, as seen in the total density profile, has a very irregular character and this is related to the specific chemical ordering of silicon and oxygen atoms in a tetrahedral network. In the layers that are closest to the walls the tetrahedra prefer an orientation such that three oxygen atoms of each tetrahedron form the first layer, the silicon atoms the second one and the remaining fourth oxygen atoms of the tetrahedra the third one (see Fig. 1).

It has been demonstrated in recent simulation studies of free silica surfaces [30, 31] that the ring distribution function is a quantity that is well suited to characterize the differences of the surface structure on intermediate length scales from that in the bulk. This holds also true in the present case: The relatively high probability of small and large rings makes the difference of the melt structure at the wall from that of the bulk. Perpendicular to the first wall layer there is a high probability of rings of size $n=3,4$ whereby, e.g., a ring with $n=3$ is typically formed by two silicon atoms from the first silicon layer and one from the second one (of course, these rings are also decorated with oxygen atoms). Parallel to the walls, at least for temperatures $T \leq 3760 \mathrm{~K}$ a relatively high probability for rings of length $n \geq 9$ is found in the wall layers which can be seen as the complement to the occurrence of small-membered rings with $n=3,4$. Our findings for the structure of the silica melt near the wall can be expected to be generic if the wall- $\mathrm{SiO}_{2}$ interactions are, as in our case, relatively weak, i.e. no covalent bonds of the wall atoms with those of the fluid are formed as it would be the case, e.g., for an interface between crystalline and amorphous silica.

Under shear, the bulk structure of the silica melt does not change for the field strengths considered in this work, $a_{\mathrm{e}} \leq 9.6 \AA / \mathrm{ps}^{2}$. This is an indication that it is justified to treat the melt as a Newtonian fluid. At the walls, the behavior of the fluid is different: If a slip motion occurs there are strong rearrangements in the ring distribution as compared to the equilibrium case. Rings of medium size break and, as a result of this, small and large rings are formed (the same effect is achieved by a local increase of the temperature). Thus, we see a rather unusual mechanism for slip motion: Slip seems to occur only if the external (gravitational-like) force field is strong enough to break rings of medium length. This slip mechanism can be expected to be generic for liquids with a tetrahedral order (of course, as before, only if walls are present that do not have a (strong) covalent bonding with the particles in the tetrahedral network).

As expected from hydrodynamic theory of a Newtonian fluid we find parabolic velocity profiles in the bulk region, although the latter region extends in the systems considered in this work only over about $15 \AA$. The values of the shear viscosity $\eta$ that we have extracted from the profiles are in fair agreement with the "Green-Kubo" values from a bulk simulation. So the question arises whether the non-equilibrium molecular dynamics technique used in this work offers a method to estimate transport coefficients. It has been demonstrated for a lattice gas model [52 that non-equilibrium simulation techniques can be very powerful to gain insight into the understanding of transport processes in binary mixtures. We have shown in this work that with the present simulations this is possible for melts with a viscosity up to about 1 Poise. For systems with higher viscosities (at lower temperatures) a different wall model would be helpful with which the tendency for slip motion is smaller and furthermore, larger system sizes would be required. But for larger system sizes one needs 
for the computation of the quasi-two-dimensional Coulomb forces faster methods than the Parry-Ewald method used in this work. A promising new method [26,27] (also based on Ewald sums) that scales with the number of particles like $N \log N$ has been proposed very recently and could be applied to systems like the one considered in this work.

Acknowledgments: The authors are grateful to W. Kob for very valuable discussions and ideas. We also thank F. Varnik and M. Müser for constructive hints and C. Mischler for a critical reading of the manuscript. Furthermore we acknowledge partial financial support from the D.I.P. Project 352-101 and we thank the HLRZ Stuttgart for a generous grant of computer time on the Cray T3E. 


\section{REFERENCES}

[1] D. J. Evans and G. P. Morris, Statistical Mechanics of Nonequilibrium Liquids (Academic, London, 1990).

[2] S. Hess, C. Aust, L. Bennett, M. Kröger, C. P. Borgmeyer, and T. Weider, Physica A 240, 126 (1997).

[3] N. V. Churaev, Liquid and Vapour Flows in Porous Bodies (Gordon and Breach, Amsterdam, 2000).

[4] M. H. Müser, Comput. Phys. Comm. 146, 54 (2002).

[5] M. H. Müser, M. H. Robbins, and M. Urbakh, Adv. Chem. Phys. (submitted).

[6] P. A. Thompson and S. M. Troian, Nature 389, 360 (1997).

[7] B. D. Todd and D. J. Evans, J. Chem. Phys. 103, 9804 (1995).

[8] K. P. Travis, B. D. Todd, and D. J. Evans, Phys. Rev. E 55, 4288 (1997).

[9] K. P. Travis and K. E. Gubbins, J. Chem. Phys. 112, 1984 (2000).

[10] J.-L. Barrat and L. Bocquet, Phys. Rev. Lett. 82, 4671 (1999).

[11] J.-L. Barrat and L. Bocquet, Faraday Discussions 112, 119 (1999).

[12] A. Onuki, R. Yamamoto, and T. Taniguchi, J. Phys. II 7, 295 (1997).

[13] R. Yamamoto and A. Onuki, J. Phys.: Condensed Matter 12, 6323 (2000).

[14] M. Kröger, W. Loose, and S. Hess, J. Rheol. 37, 1057 (1993).

[15] M. Kröger and S. Hess, Phys. Rev. Lett. 85, 1128 (2000).

[16] C. Aust, M. Kröger, and S. Hess, Macromolecules 32, 5660 (1999).

[17] T. Kreer, M. H. Müser, K. Binder, and J. Klein, Langmuir 17, 7804 (2001).

[18] F. Varnik and K. Binder, Phys. Rev. E, in press.

[19] P. H. Poole, P. F. McMillan, and G. H. Wolf, Rev. in Mineralogy 32, 563 (1995).

[20] D. E. Parry, Surf. Sci. 49, 433 (1975).

[21] D. E. Parry, Surf. Sci. 54, 195 (1976).

[22] D. M. Heyes, Phys. Rev. B 49, 755 (1994).

[23] S. W. de Leeuw and J. W. Perram, Physica A 113A, 546 (1982).

[24] A. H. Widmann and D. B. Adolf, Comput. Phys. Commun. 107, 167 (1997).

[25] R. Strebel and R. Sperb, Mol. Simul. 27, 61 (2001).

[26] A. Arnold, J. de Joannis, and C. Holm, J. Chem. Phys. 117, 2496 (2002).

[27] J. de Joannis, A. Arnold, and C. Holm, J. Chem. Phys. 117, 2503 (2002).

[28] O. V. Mazurin, M. V. Streltsina, and T. P. Shvaiko-Shvaikovskaya, Handbook of Glass Data, Part A: Silica Glass and Binary Silicate Glasses (Elsevier, Amsterdam, 1983).

[29] D. Frenkel and B. Smit, Understanding Molecular Simulation (Academic, San Diego, 1996).

[30] A. Roder, W. Kob, and K. Binder, J. Chem. Phys. 114, 7602 (2001).

[31] C. Mischler, W. Kob, and K. Binder, Comp. Phys. Comm., in press (2002).

[32] J.-F. Poggemann, A. Go, G. Heide, E. Rädlein, and G. H. Frischat, J. Non-Cryst. Solids 281, 221 (2001).

[33] L. Bocquet and J.-L. Barrat, Phys. Rev. E 49, 3079 (1994).

[34] V. P. Sokhan, D. Nicholson, and N. Quirke, J. Chem. Phys. 115, 3878 (2001).

[35] J.-P. Hansen and I. R. McDonald, Theory of Simple Liquids (Academic, London, 1986).

[36] J. Horbach and W. Kob, Phys. Rev. B 60, 3169 (1999).

[37] B. W. H. van Beest, G. J. Kramer, and R. A. van Santen, Phys. Rev. Lett. 64, 1955 (1990). 
[38] K. Vollmayr, W. Kob, and K. Binder, Phys. Rev. B 54, 15808 (1996).

[39] T. Koslowski, W. Kob, and K. Vollmayr, Phys. Rev. B 56, 9469 (1997).

[40] S. N. Taraskin and S. R. Elliott, Phys. Rev. B 56, 8605 (1997).

[41] J. Horbach, W. Kob, and K. Binder, J. Phys. Chem. B 103, 3104 (1999).

[42] J. Horbach, W. Kob, and K. Binder, Eur. Phys. J. B 19, 531 (2001).

[43] P. Scheidler, W. Kob, A. Latz, J. Horbach, and K. Binder, Phys. Rev. B 63, 104204 (2001).

[44] P. Jund and R. Jullien, Phys. Rev. B 59, 13707 (1999).

[45] M. Benoit, S. Ispas, P. Jund and R. Jullien, Eur. Phys. J. B 13, 631 (2000).

[46] S. Nosè and M. Klein, Mol. Phys. 50, 1055 (1983).

[47] S. Nosè, J. Chem. Phys. 81, 511 (1984).

[48] W. Hoover, Phys. Rev. A 31, 1695 (1985).

[49] Here and in the following only symmetrized profiles are shown.

[50] see, e.g., J. F. Stebbins, Rev. Mineral. 32, 191 (1995) and references therein.

[51] J. Koplik and J. R. Banavar, Phys. Rev. Lett. 80, 5125 (1998).

[52] K. W. Kehr, K. Binder, and S. Reulein, Phys. Rev. B 39, 4891 (1989). 


\section{FIGURES}

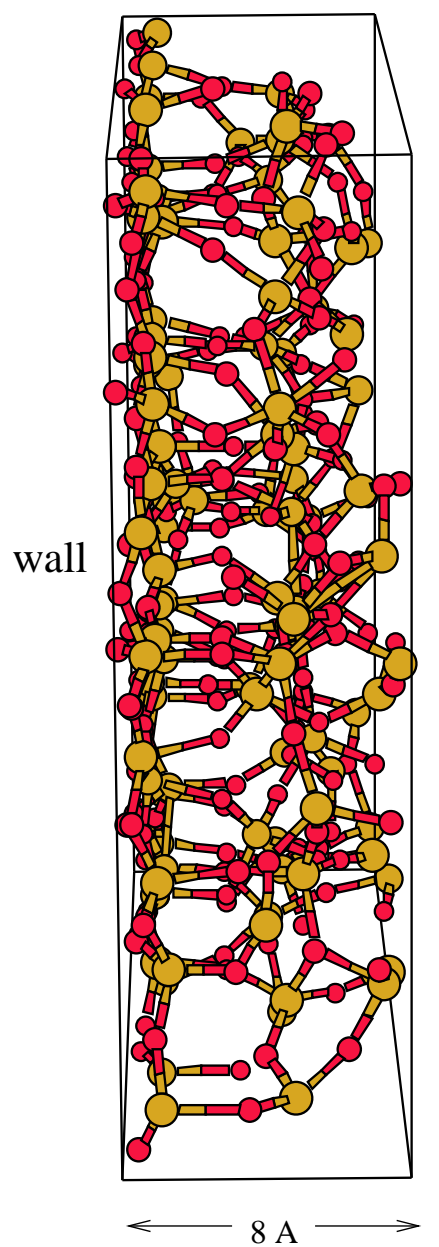

FIG. 1. Snapshot of a part of a configuration at $T=3760 \mathrm{~K}$ that is within $8 \AA$ away from the wall. The brown and the red spheres correspond to the silicon and oxygen atoms, respectively. 

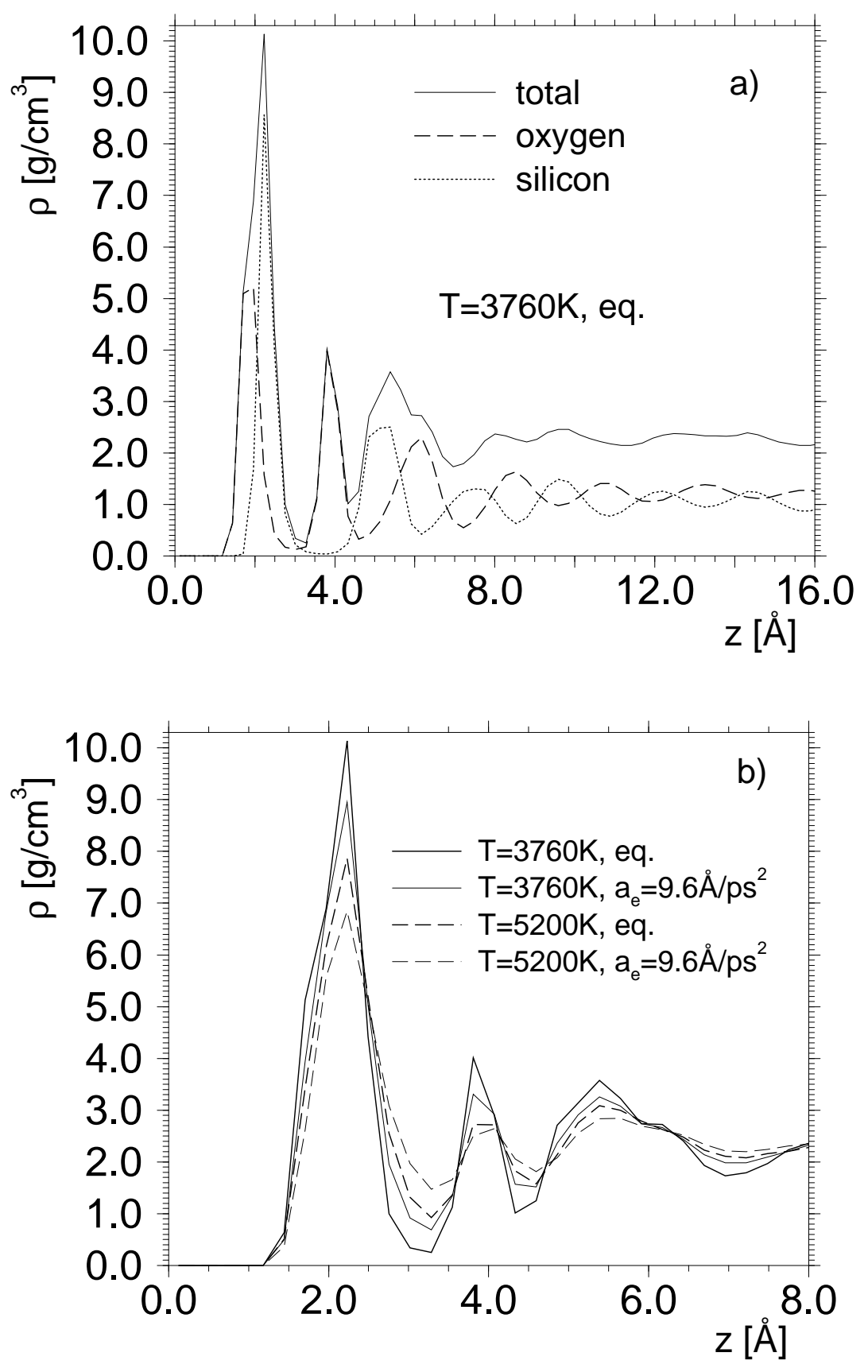

FIG. 2. a) Total density profile and partial density profiles for oxygen and silicon at $T=3760 \mathrm{~K}$. b) Total density profiles at equilibrium and with acceleration field $a_{\mathrm{e}}=9.6 \AA / \mathrm{ps}^{2}$ for the two temperatures $T=3760 \mathrm{~K}$ and $T=5200 \mathrm{~K}$. 

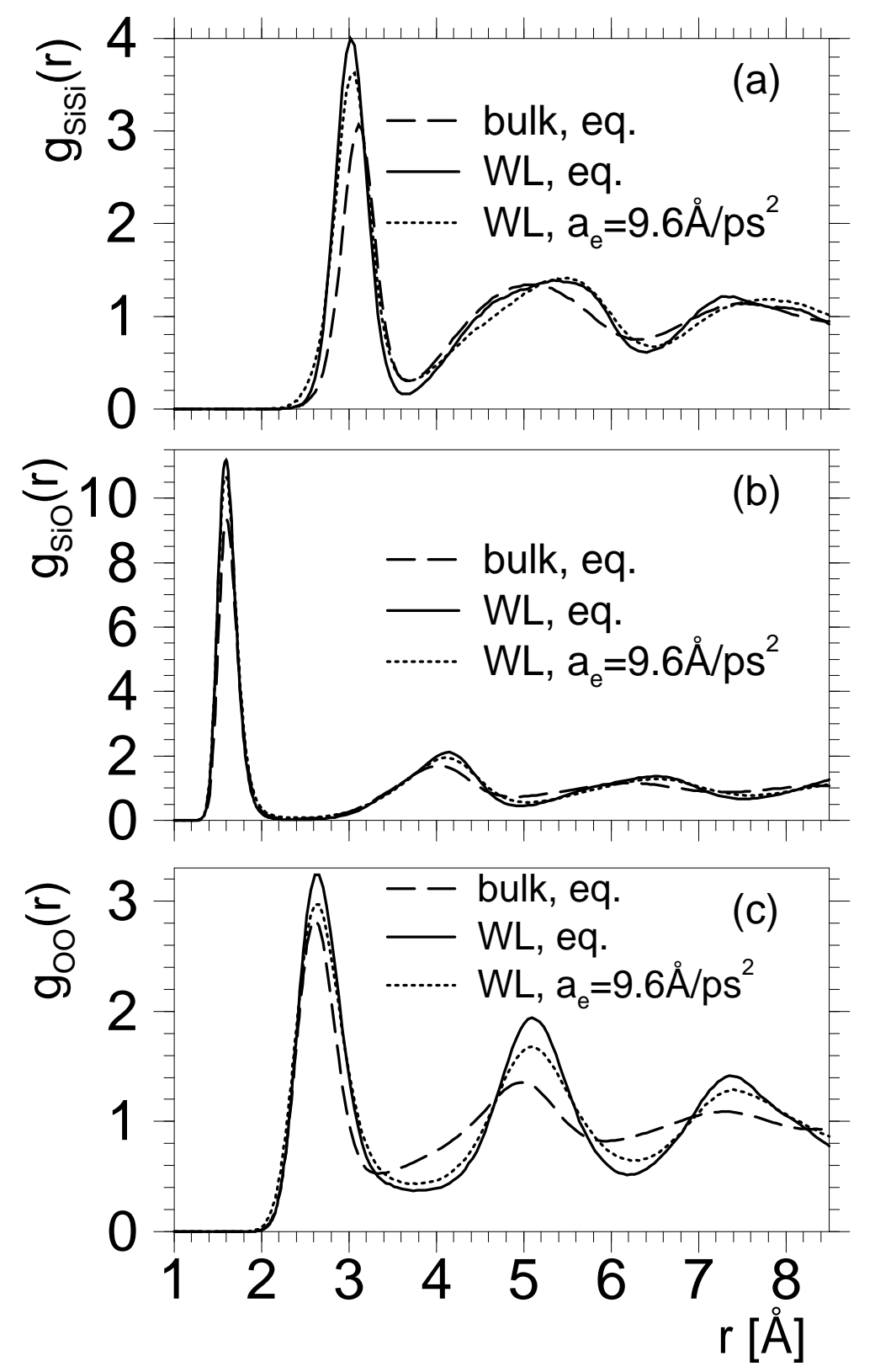

FIG. 3. Partial radial distribution functions $g_{\alpha \beta}(r)$ at equilibrium in the bulk and in the WL, and with the field $a_{\mathrm{e}}=9.6 \AA / \mathrm{ps}^{2}$ in the WL. The temperature is $T=3760 \mathrm{~K}$. a) $\left.g_{\mathrm{SiSi}}, \mathrm{b}\right) g_{\mathrm{SiO}}, \mathrm{c}$ ) $g_{\mathrm{OO}}$. 

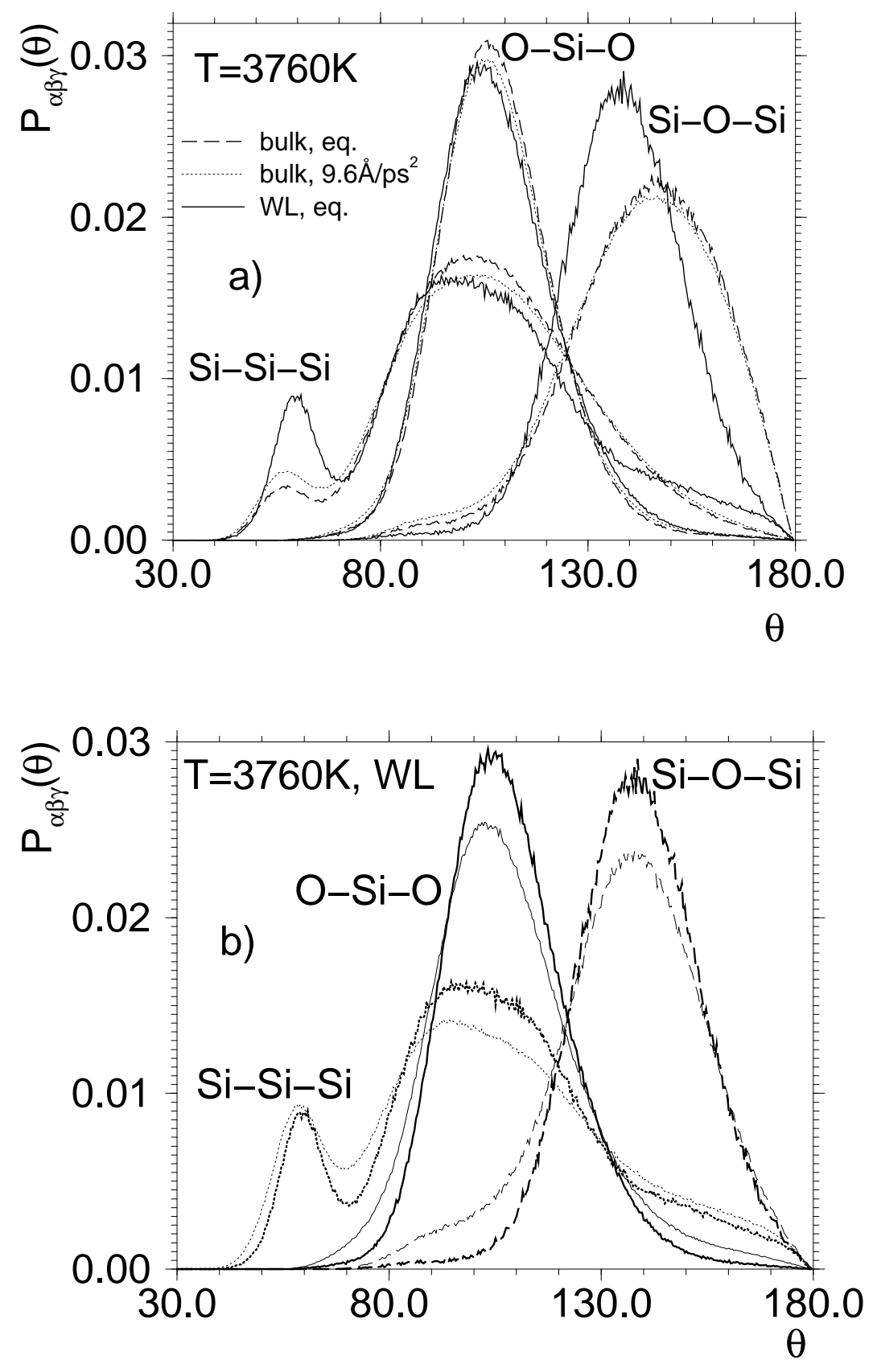

FIG. 4. Distributions of the indicated angles at equilibrium and with external field $a_{\mathrm{e}}=9.6 \AA / \mathrm{ps}^{2}$ for $T=3760 \mathrm{~K}$. a) Bulk and WL (at equilibrium), b) WL at equilibrium (bold lines) and with external field (thin lines). 

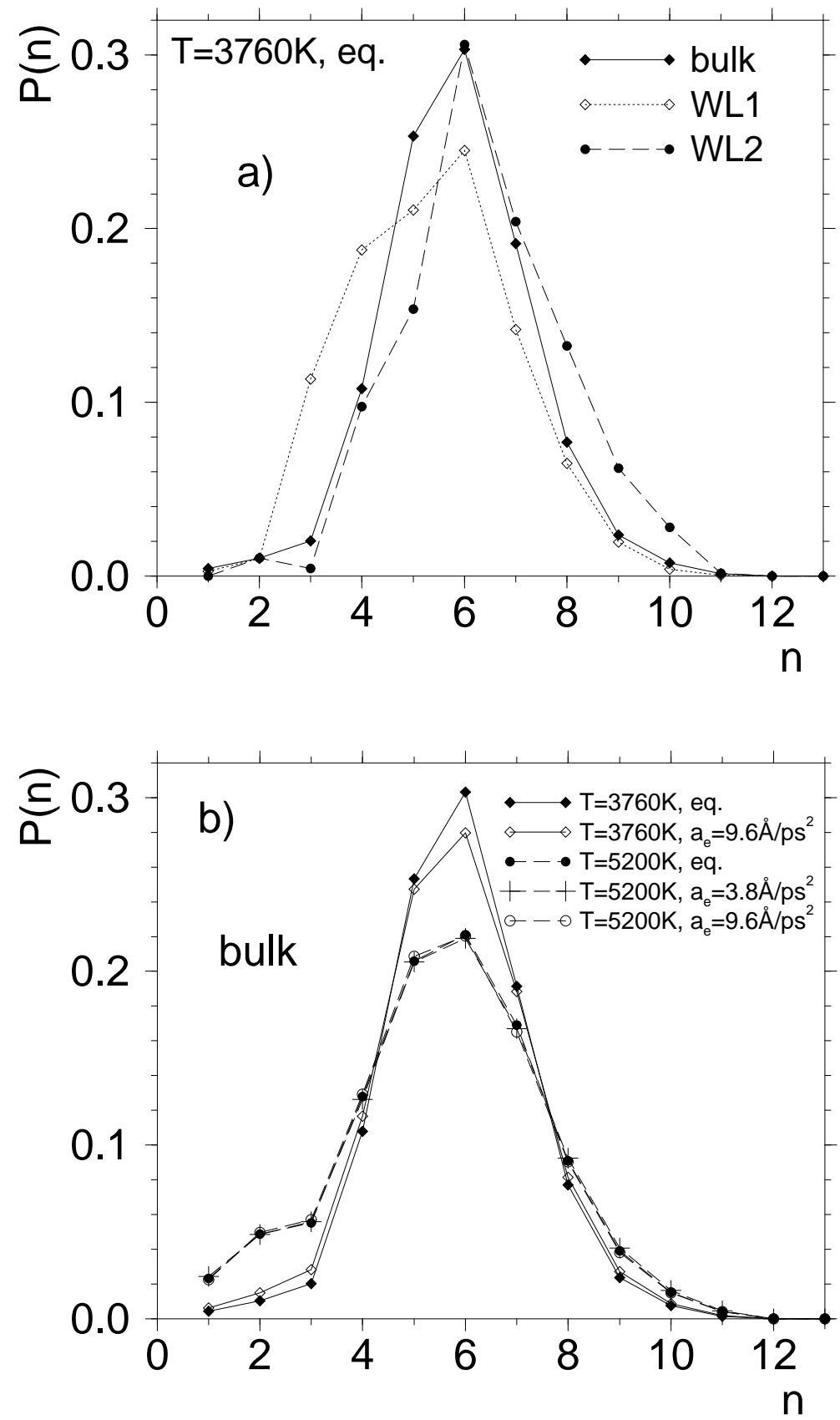

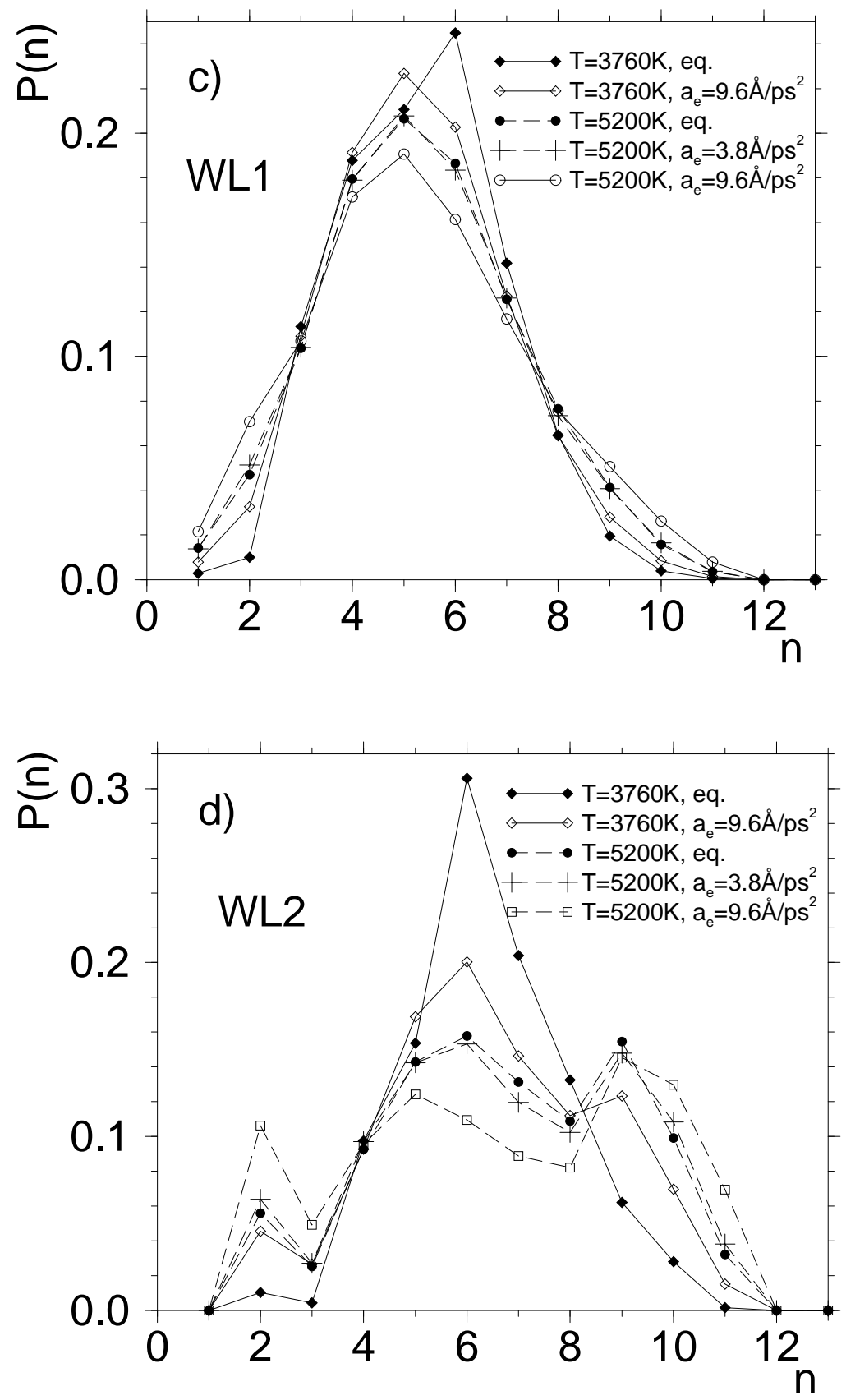

FIG. 5. Probability $P(n)$ that a ring has a length $n$, a) at $T=3760 \mathrm{~K}$ in equilibrium for the bulk, WL1, and WL2, b) at $T=3760 \mathrm{~K}$ and $T=5200 \mathrm{~K}$ in equilibrium and under the indicated acceleration fields in the bulk, c) the same as in b) but for WL1, and d) also the same as in b) but for WL2. For the definition of WL1 and WL2 see text. 


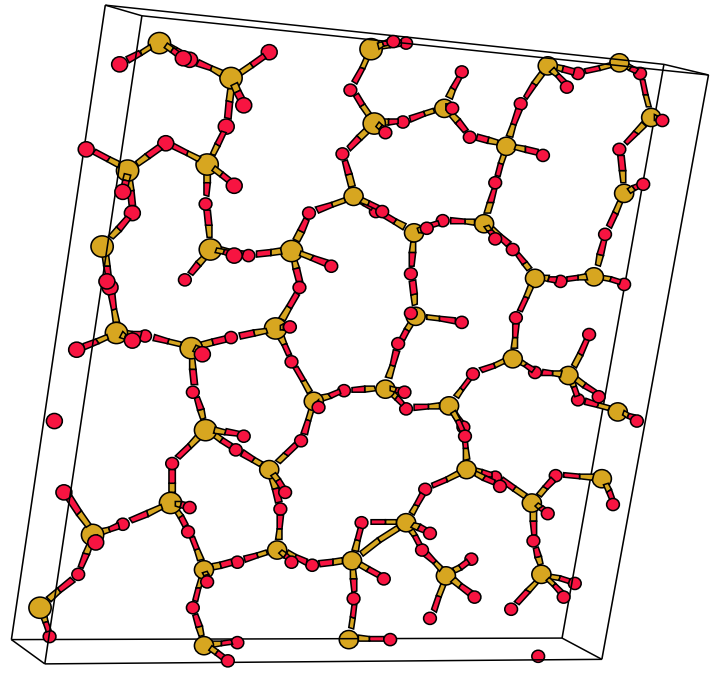

a)

FIG. 6. Snapshot of the wall layers (WL2) for $T=3760 \mathrm{~K}$, a) at equilibrium and b) with the acceleration field $a_{\mathrm{e}}=9.6 \AA / \mathrm{ps}^{2}$ (the direction of $a_{\mathrm{e}}$ is from the left to the right). The dark and the light grey spheres correspond to the silicon and oxygen atoms, respectively.

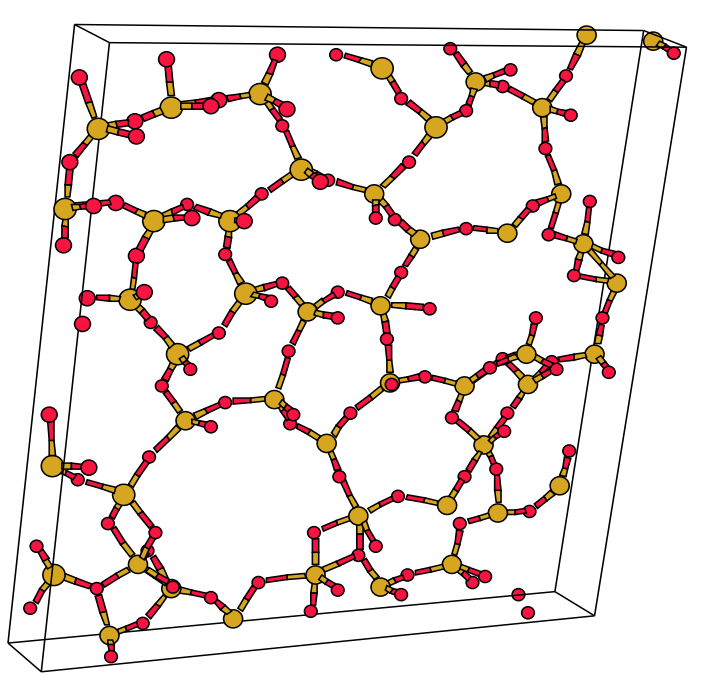

b) 


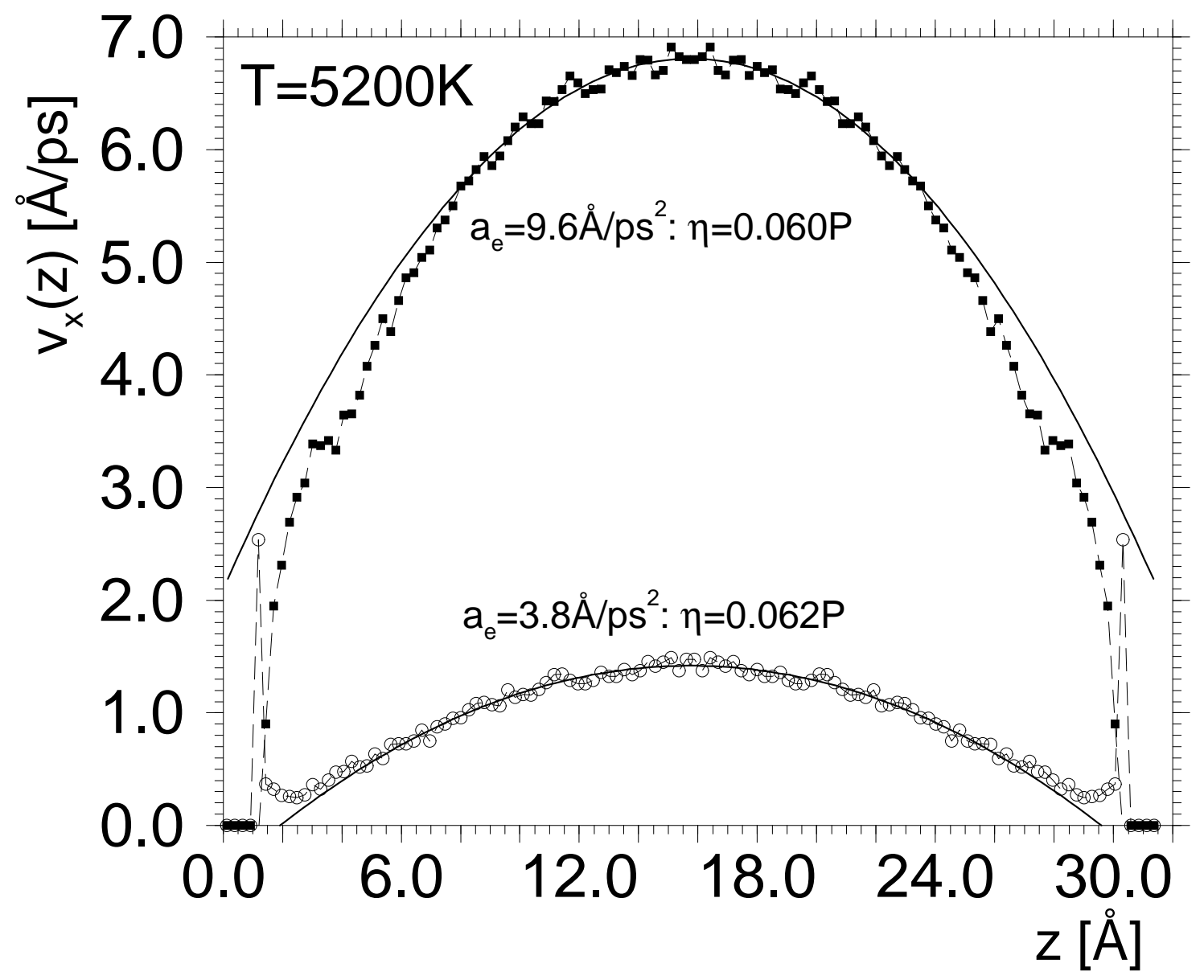

FIG. 7. Velocity profiles at $T=5200 \mathrm{~K}$ for the indicated acceleration fields. The bold lines are fits with formula (3) and the indicated values for the shear viscosity $\eta$ are extracted from these fits. 


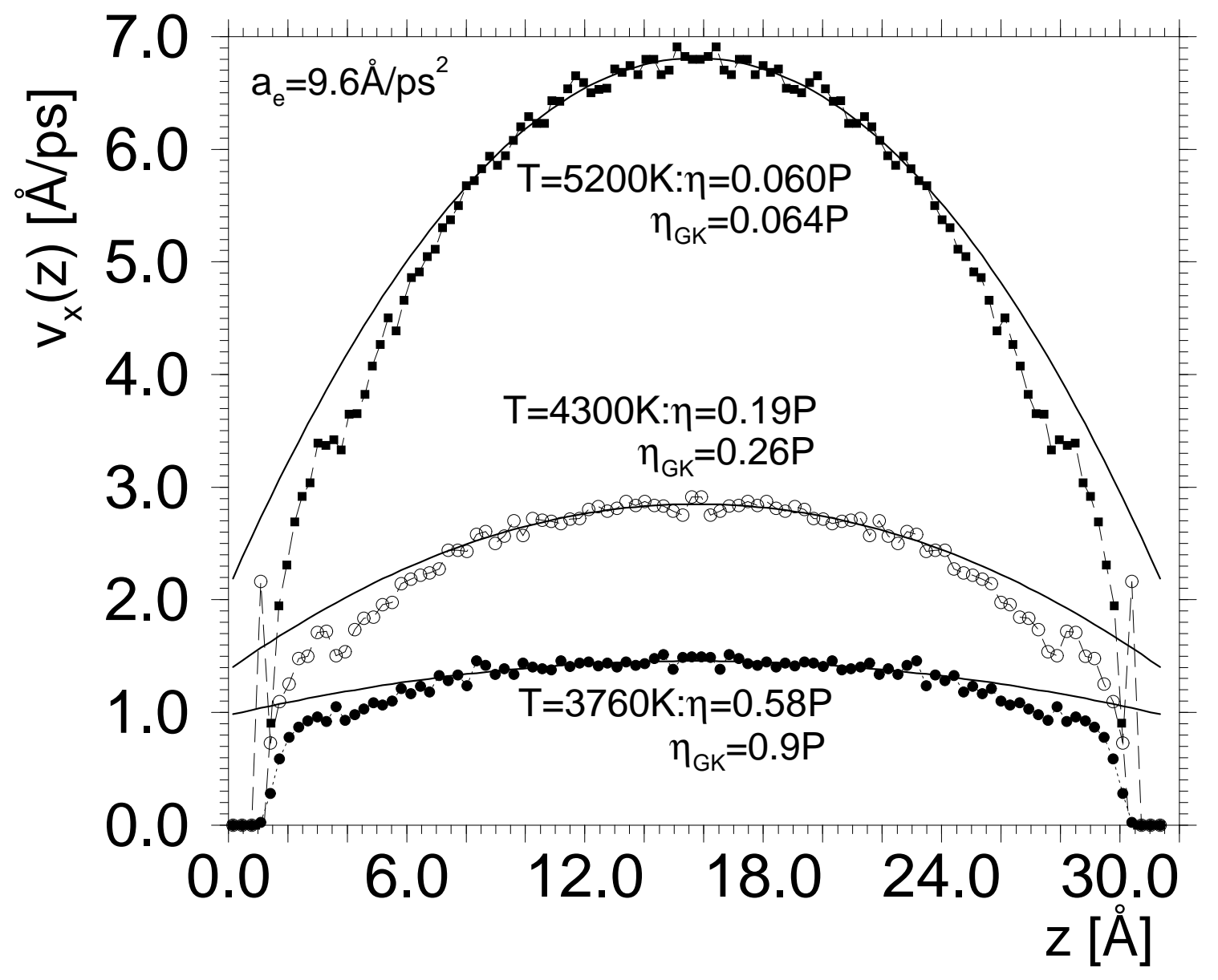

FIG. 8. Velocity profiles for $a_{\mathrm{e}}=9.6 \AA / \mathrm{ps}^{2}$ at different temperatures. $\eta_{\text {GK }}$ denotes the "Green-Kubo" results that are taken from Horbach and Kob [36]. The rest is similar to Fig. 0. 